\title{
Relocation of Resonance Frequencies of Undamped Systems Adding Grounded Springs
}

\author{
Orhan ÇAKAR Mesut Hüseyinoğlu
}

\begin{abstract}
Relocation of the resonance frequencies of the un-damped systems to desired values adding grounded springs is addressed. For the calculation of the necessary springs' stiffnesses, a method based on Sherman-Morrison (SM) formula is presented. The method uses receptances of the original system and it needs neither analytical nor modal models. Numerical simulation shows that the method is very effective.
\end{abstract}

Keywords-natural frequency, receptance, inverse modification, Sherman-Morrison formula

\section{Introduction}

Structural modification is mostly made to avoid resonance phenomenon. In general there are two kinds of structural modifications. First, direct structural modification is the estimation of the dynamic properties of an existing structure after changing the physical properties such as mass, stiffness and damping. Second, inverse structural modification is the estimation of the modifications needed to satisfy the desired dynamic properties. Inverse structural modification problems are rather difficult, since the solution is usually not unique and it is mostly needed to solve the nonlinear equations set with sensitivity analysis.

Structural modification methods are based on the use of modal properties e.g. [1-3] or response properties e.g. [4-8]. These properties can be determined from finite element (FE) or experimental modal analysis (EMA). In applications, the use of frequency response functions (FRFs) derived from EMA is preferred, because these methods don't need the physical models.

Shifting natural frequencies has been studied by a lot of researchers. Tsuei and Yee [9] presented a method based on the force response of the original dynamic system such that it provides the sensitivity and feasibility of shifting the eigen-frequency to a desired value. Ram [10] presented a strategy for enlarging a spectral gap by adding two appropriate oscillators at the proper locations. McMillan and Keane [11] studied on shifting resonance from a frequency band applying concentrated masses to a plate. Mottershead and his coworkers [12-15] presented a series of papers on assigning modes to the dynamical systems.

Orhan ÇAKAR,

Firat University,Department of Mechanical Eng.

Elazig/TURKEY
Farahani and Bahai [16] proposed an inverse strategy for the relocation of structural natural frequencies. In the proposed method, a sensitivity analysis of the systems eigenvalues with respect to material or geometrical parameters of the structure is conducted. The required parameter variation to achieve a desired frequency shift for the structure is then computed. Lawther [17] presented a comprehensive study on removing frequencies from a given range changing the stiffness. The success of the method was assessed according to both rank of changes, and the number of freedoms that they connect to.

Recently, Ouyang and his coworkers [18-21] studied the passive structural modifications of the mass-spring systems for assignment of the eigen-structures i.e. eigenvalues and eigen-vectors. Except one, all of the proposed methods in these studies use the receptances.

In this study, a method is presented in order to relocate some specified resonance frequencies to desired values by adding grounded springs without changing the degree of freedom of the systems. The method is based on the SM formula and it estimates the necessary stiffnesses of springs using a number of receptances related to modification coordinates of the system.

\section{Structural Modification Using SM Formula}

If $[A]^{-1}$ is the inverse of a non-singular square matrix $[A]$ and the modification is expressed as a product of two vectors such as $\{u\}\{v\}^{T}$, so that the modified matrix is given by

$$
\left[A^{*}\right]=[A]+\{u\}\{v\}^{T}
$$

the inverse of the modified matrix $\left[A^{*}\right]^{-1}$ can be calculated by using the SM formula as [22]:

$$
\left[A^{*}\right]^{-1}=[A]^{-1}-\frac{\left([A]^{-1}\{u\}\right)\left(\{v\}^{T}[A]^{-1}\right)}{1+\{v\}^{T}[A]^{-1}\{u\}}
$$

The SM formula given by (2), has been used in a wide variety of applications in the past [23-25].

The relation of the SM formula with structural modification can be explained as follows. It is known that the dynamic stiffness matrix $[Z]$ of a system is given by

$$
[Z]=[K]-\omega^{2}[M]+j \omega[C]
$$


where $[K],[M],[C]$ represent stiffness, mass and damping matrices, $\omega$ is the angular frequency and $j=\sqrt{ }-1$. Let $[\Delta Z]$ be the modification matrix which includes the mass, stiffness and damping modifications, using the well-known relationship between the receptance and the dynamic stiffness, $[\alpha]=[Z]^{-1}$, and expressing the modified system as $\left[Z^{*}\right]=[Z]+[\Delta Z]$ where $[\Delta Z]=\{u\}\{v\}^{T}$, the receptances of the modified system can be computed using the SM formula in (2) as

$$
\left[\alpha^{*}\right]=\left[Z^{*}\right]^{-1}=[\alpha]-\frac{([\alpha]\{u\})\left(\{v\}^{T}[\alpha]\right)}{1+\{v\}^{T}[\alpha]\{u\}}
$$

The $\left[\alpha^{*}\right]$ matrix above contains the receptances of the modified system. If only one-rank modification is considered at co-ordinate $r$ alone, then all elements of the modification vectors $u$ and $v$ are zero except $r^{\text {th }}$ elements. For example, $u_{r}=1$ and $v_{r}=k$ for a grounded spring $(k)$ modification at the co-ordinate $r$.

Equation (4) needs all the elements of the receptance matrix $[\alpha]$ of the structure, but this is not desirable in practical applications. However, it can be written in active co-ordinates only, i.e. excitation, response and modification co-ordinates as shown by Sanliturk [26]. Furthermore, Cakar and Sanliturk [27] expressed an explicit formula for any receptance as follows:

$$
\alpha_{p q}^{*}=\frac{\alpha_{p q}+v_{r}\left(\alpha_{r r} \alpha_{p q}-\alpha_{p r} \alpha_{r q}\right)}{1+v_{r} \alpha_{r r}}
$$

where $p, q$ and $r$ represent response, excitation and modification co-ordinates, respectively. (5) is a general expression allowing the calculation of the modified receptance $\alpha_{p q}^{*}$ by using the original receptances and the modification. It should be noted that (5) can be used for more than one modification successively.

Cakar and Sanliturk [27] used the formula given in (5) for the purpose of direct structural modification, where the modification was the transducer mass. The frequency response functions (FRFs) of the structures without transducer mass were obtained by modifying the structure with negative mass. After that Çakar [28] developed (5) for the inverse structural modification, where the aim was to preserve one of the predetermined natural frequencies of a structure, by adding a grounded spring after some modifications. After adding one or more known masses to the structure, Çakar [28] developed a method for the calculation of the necessary stiffness to prevent the shifting of any one natural frequency of the structure. Further, Huseyinoğlu and Cakar [29] extended the same method by adding a spring between two co-ordinates instead of adding a grounded one.

In the present study, as a natural extension of the early studies of authors, the aim is relocating some troubled natural frequencies of a dynamic system to the desired values by adding grounded springs. Specially, two natural frequencies are considered. The method is outlined in the following section.

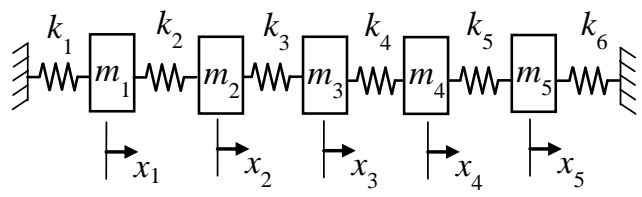

a) Original five-dof system

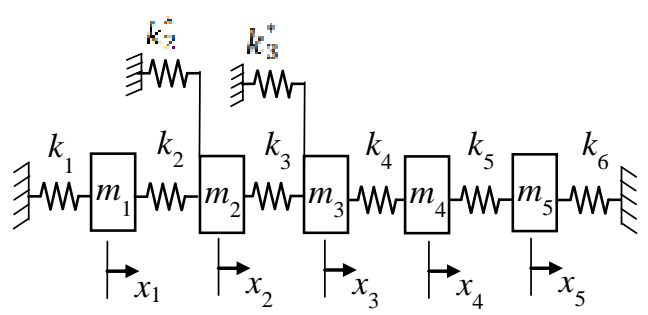

b) The system modified by two grounded springs.

Figure 1. A five dof mass-spring system. a) Original system, b) Modified system

\section{Relocation of Resonance Frequencies}

To clarify the method, consider a five-dof mass-spring system given in Fig.1a [17]. In this study, it is required that two natural frequencies of the system are relocated to desired values. In order to achieve this, two grounded springs $k_{2}^{*}$ and $k_{3}^{*}$ will be added to the system at coordinates 2 and 3 as shown in Fig. 1b. The main problem is to find these stiffnesses of the springs. It should be stated that the number of spring modifications is chosen equal to the number of frequencies relocated and the modification co-ordinates are randomly chosen here for the simplicity. Other solutions are not of interest for this study.

Supposing we have the receptances of the original system related to the modification co-ordinates, we will try to obtain receptances of the modified system. These two modifications can be made successively and the receptances of the modified system can be calculated using (5). First, add the spring $k_{2}^{*}$ to the system at co-ordinate 2 . In this case the modification co-ordinate is $r=2$ and the modification is $v_{r}=k_{2}^{*}$. The transfer receptance of the modified system can be calculated via (5) as

$$
\alpha_{23}^{*}=\frac{\alpha_{23}}{1+k_{2}^{*} \alpha_{22}}
$$

Then add the spring $k_{3}^{*}$ to the system. In this case the modification co-ordinate is $r=3$ and the modification is $v_{r}=k_{3}^{*}$. After that, the receptance of the modified system can be obtained by using (5) successively as: 
Proc. of the Fourth Intl. Conf. Advances in Civil, Structural and Mechanical Engineering- CSM 2016

Copyright (c) Institute of Research Engineers and Doctors, USA .All rights reserved. ISBN: 978-1-63248-093-4 doi: 10.15224/ 978-1-63248-093-4-35

$$
\alpha_{23}^{* *}=\frac{\alpha_{23}^{*}}{1+k_{3}^{*} \alpha_{33}^{*}}
$$

where $\alpha_{23}^{*}$ is already known from (6) and $\alpha_{33}^{*}$ can be calculated from (5) by writing first $p=3$, then $r=2, q=3$ and $v_{r}=k_{3}^{*}$ as:

$$
\alpha_{33}^{*}=\frac{\alpha_{33}+k_{2}^{*}\left(\alpha_{22} \alpha_{33}-\alpha_{23}^{2}\right)}{1+k_{2}^{*} \alpha_{22}} .
$$

It is desired to relocate two natural frequencies of the system at new values, say $\omega_{s 1}$ and $\omega_{s 2}$. This means that the receptances values of the final system must go to infinity at these frequencies, and this requires the denominator of (7) goes to zero. In this manner two nonlinear equations with two unknowns are obtained as follows:

$$
\begin{aligned}
& \alpha_{23}^{* *}\left(\omega_{s 1}\right) \rightarrow \infty \Rightarrow 1+k_{3}^{*} \alpha_{33}^{*}\left(\omega_{s 1}\right)=0 \\
& \alpha_{23}^{* *}\left(\omega_{s 2}\right) \rightarrow \infty \Rightarrow 1+k_{3}^{*} \alpha_{33}^{*}\left(\omega_{s 2}\right)=0
\end{aligned}
$$

By solving the above nonlinear equations set numerically the values of spring coefficients $k_{2}^{*}$ and $k_{3}^{*}$ can be obtained.

\section{Numerical Simulation}

For the numerical verification of the method, the springmass system given in Fig. 1 is considered again. The coefficients of springs are 1 unit and the masses are 1, 2, 3, 2 and 1 units respectively (same as given in [17]). The natural frequencies and related normalized mode shapes of the original system are given in Table I.

For this simulation it is desired to relocate the two natural frequencies at $0.10 \mathrm{~Hz}$ and $0.15 \mathrm{~Hz}$. To achieve this, two grounded springs $k_{2}^{*}$ and $k_{3}^{*}$ will be added at co-ordinates 2 and 3 as shown in Fig. 1b. First the receptances of the original system are calculated and three of them are given in Fig.2. After substituting the values of the receptances at 0.10 $\mathrm{Hz}$ and $0.15 \mathrm{~Hz}$ into (8) several two nonlinear equations are found in (9). Finally, the stiffnesses of these springs, $k_{2}^{*}$ and $k_{3}^{*}$, are obtained by solving these two nonlinear equations numerically. A MATLAB code was implemented to manage these mathematical procedures. Table II shows the natural frequencies desired to relocate and the calculated stiffnesses of the springs.

TABLE I. NATURAL FREQUENCIES AND MOdE SHAPES OF ORIGINAL SYSTEM

\begin{tabular}{|c|c|c|c|c|c|}
\hline \multirow{2}{*}{} & \multicolumn{5}{|c|}{ Modes } \\
\cline { 2 - 6 } & $\mathbf{1}$ & $\mathbf{2}$ & $\mathbf{3}$ & $\mathbf{4}$ & $\mathbf{5}$ \\
\hline $\boldsymbol{\omega}_{\boldsymbol{n}}(\boldsymbol{H z})$ & 0.0556 & 0.1267 & 0.1695 & 0.2448 & 0.2471 \\
\hline & 0.1769 & -0.3251 & -0.3235 & -0.6280 & 0.6034 \\
& 0.4698 & -0.6280 & -0.3959 & 0.3251 & -0.3500 \\
& 0.7042 & -0.0000 & 0.6908 & 0.0000 & 0.1639 \\
& 0.4698 & 0.6280 & -0.3959 & -0.3251 & -0.3500 \\
& 0.1769 & 0.3251 & -0.3235 & 0.6280 & 0.6034 \\
\hline
\end{tabular}

TABLE II. NatURAL FReQuENCIES DesiRED To Relocate AND

\begin{tabular}{|l|c|c|}
\multicolumn{4}{|c|}{ CALCULATED SPRINGS's STIFFNESSES } \\
\hline \multirow{2}{*}{ Desired frequencies (Hz) } & $\boldsymbol{\omega}_{\mathbf{s} 1}$ & $\omega_{\mathbf{s} 2}$ \\
\cline { 2 - 3 } & 0.10 & 0.15 \\
\hline \multirow{2}{*}{ Calculated springs (unit) } & $k_{2}^{*}$ & $k_{3}^{*}$ \\
\cline { 2 - 3 } & 1.113 & 1.475 \\
\hline
\end{tabular}
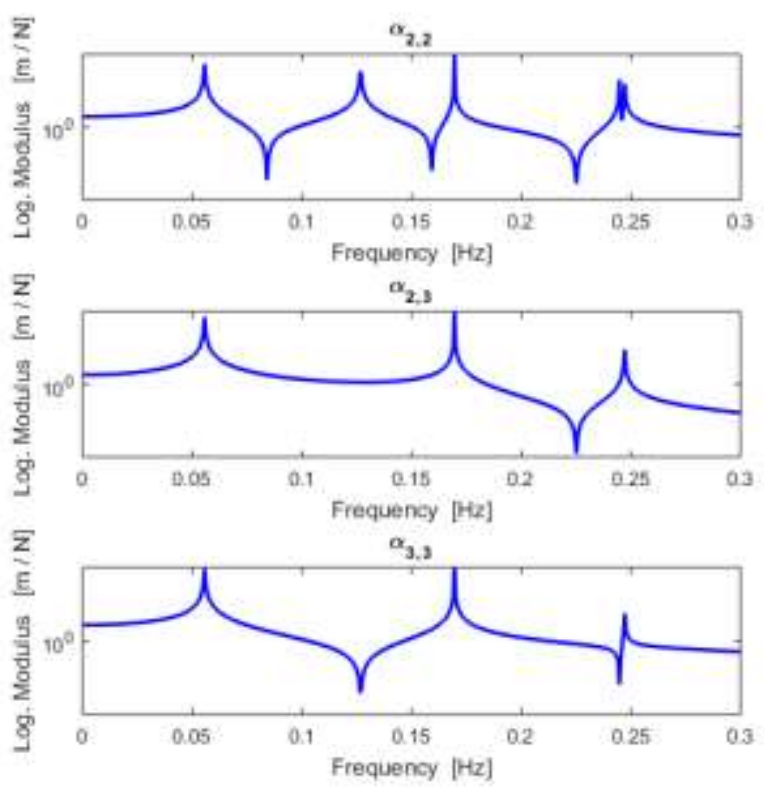

Figure 2. Receptances of the original system.

After adding the calculated springs to the system, the natural frequencies and mode shapes of the modified system are determined by solving an eigenvalue problem and are given in Table III. As shown in Table III the first two natural frequencies of the new (modified) system are 0.10 $\mathrm{Hz}$ and $0.15 \mathrm{~Hz}$ which match exactly desired frequencies values. Also the receptances of the modified system are calculated and compared to those of original system in Fig. 3. This result shows the efficiency of the proposed method.

Note that, the first two natural frequencies of the system shift to new values since the grounded springs do not change the freedom of system.

TABLE III. NATURAL Frequencies AND Mode ShaPes OF ModifiEd SYSTEM

\begin{tabular}{|c|c|c|c|c|c|}
\hline \multirow{2}{*}{} & \multicolumn{5}{|c|}{ Modes } \\
\cline { 2 - 6 } & $\mathbf{1}$ & $\mathbf{2}$ & $\mathbf{3}$ & $\mathbf{4}$ & $\mathbf{5}$ \\
\hline \multirow{2}{*}{$\boldsymbol{\omega}_{\boldsymbol{n}}(\boldsymbol{H z})$} & $\mathbf{0 . 1 0 0 0}$ & $\mathbf{0 . 1 5 0 0}$ & 0.1930 & 0.2460 & 0.2553 \\
\hline & 0.1118 & -0.4215 & -0.4656 & 0.1953 & 0.7449 \\
& 0.2537 & -0.6627 & -0.3487 & -0.1075 & -0.6028 \\
& 0.5283 & -0.3546 & 0.7329 & -0.1191 & 0.2094 \\
& 0.7344 & 0.4282 & -0.2115 & 0.4665 & -0.1224 \\
& 0.3235 & 0.2723 & -0.2824 & -0.8477 & 0.1513 \\
\hline
\end{tabular}




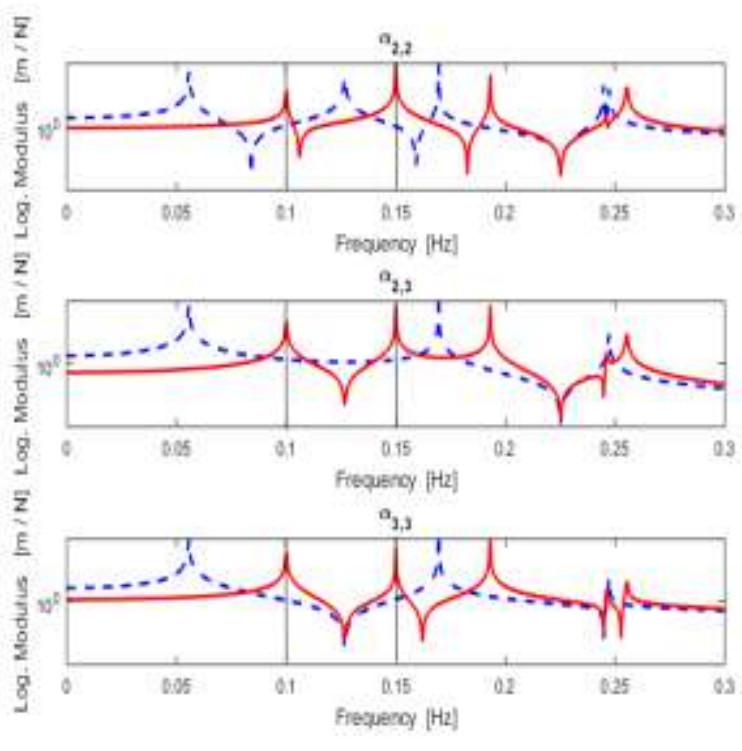

Figure 3. Receptances of the original (--) and modified (-) systems.

\section{v. Conclusion}

The phenomenon of resonance is one of the most important problems in engineering. One way to avoid resonance is to shift troublesome natural frequencies of the dynamic system away from the frequency of the exciting force. This requires modifying the physical parameters of the system. In this study, the relocation of two resonance frequencies of the undamped systems to given values by adding two grounded springs was investigated. An inverse modification method based on SM formula was developed to calculate the necessary stiffnesses of the springs. Numerical simulation shows that this method is very effective. One of the advantages of the method is that it uses the receptances of the original system therefore the physical model is not needed.

It should be noted that the solution is not unique due to nature of the inverse problem. In the case of choosing different co-ordinates for the addition of springs a different solution is found. On the other hand, in this study the number of modifications chosen is equal to the number of frequencies relocated which is two. This is a minimum requirement to solve the problem. For the relocation of two frequencies, more than two modifications may be chosen. Also different modifications, including masses and the stiffnesses of the system, may be made. These problems are of interest to authors, who have studied on to relocate more than two resonance frequencies and their appropriate modifications.

\section{References}

[1] I. Bucher, and S. Braun, "The Structural modification inverse problem: An exact solution", Mechanical Systems and Signal Processing, 1993, vol. 7(3), pp. 217-238.

[2] D.D. Sivan, and Y. M. Ram, "Mass and Stiffness Modifications to Achieve Desired Natural Frequencies", Comm. in Numerical Methods in Engineering, 1996, vol. 12, pp. 531-542.
[3] S. G. Braun, and Y. M. Ram, "Modal Modification of Vibrating Systems: Some Problems and Their Solutions", Mechanical Systems and Signal Processing, 2001, vol. 15(1), pp. 101-119.

[4] H. N. Özgüven, "Structural Modifications Using Frequency Response Functions", Mechanical Systems and Signal Processing, 1990, vol. 4(1), pp. 53-63.

[5] K.J. Chang, and Y.P. Park, "Substructural Dynamic Modification using Component Receptance Sensitivity", Mechanical Systems and Signal Processing, vol.12, pp.525-541, 1998.

[6] L. Tao, and H. Jimin, "Local structural modification using mass and stiffness changes", Engineering Structures, vol.21(11), pp.1028-1037, 1999.

[7] Y.H. Park, and Y.S. Park, "Structural Modification Based on Measured Frequency Response Functions: An Exact Eigenproperties Reallocation", Journal of Sound and Vibration, vol.237(3), 411-426, 2000.

[8] Y.M. Ram, "Dynamic Structural Modification. The Shock and Vibration Digest, 2000, vol.32(1), pp. 11-17.

[9] Y. G. Tsuei and E. K. L. Yee, "A method for modifying dynamic properties of undamped mechanical systems", ASME Journal of Dynamic Systems, Measurement and Control”, vol.111, pp.403-408, 1989.

[10] Y.M. Ram, "Enlarging a spectral gap by structural modification", Journal of Sound and Vibration, vol.176(2), pp.225-234, 1994.

[11] J. McMillan, and A. J. Keane, "Shifting resonances from a frequency band by applying concentrated masses to a thin rectangular plate", Journal of Sound and Vibration, vol.192 (2), pp.549-562, 1996,.

[12] J. E. Mottershead and G. Lallement, "Vibration Nodes, and the Cancellation of Poles and Zeros by Unit-Rank Modifications to Structures", Journal of Sound and Vibration, vol.222(5), pp.833-851, 1999.

[13] J. E. Mottershead, "Structural Modification for the Assignment of Zeros Using Measured Receptances", ASME Journal of Applied Mechanics, vol. 68, pp. 791-798, 2001.

[14] J. E. Mottershead, C. Mares, and M. I. Friswell, "An inverse method for the assignment of vibration nodes", Mechanical Systems and Signal Processing, vol.15 (1), pp.87-100, 2001.

[15] A. Kyprianou, JE. Mottershead and H. Ouyang, "Assignment of natural frequencies by an added mass and one or more springs", Mechanical Systems and Signal Processing, vol.18, pp.263-289,2004.

[16] K. Farahani, H. Bahai, "An inverse strategy for relocation of eigenfrequencies in structural design. Part I: first order approximate solutions", Journal of Sound and Vibration, vol.274, pp.481-505, 2004

[17] R. Lawther, "Assessing how changes to a structure can create gaps in the natural frequency spectrum", International Journal of Solids and Structures, vol.44, pp.614-635, 2007.

[18] H. Ouyang, D. Richiedei, A. Trevisani , G. Zanardo, "Eigenstructure assignment in undamped vibrating systems: a convex-constrained modification method based on receptances", Mechanical Systems and Signal Processing, vol.27 (2), pp.397-409, 2012.

[19] H. Ouyang, D. Richiedei, A. Trevisani , G. Zanardo, "Discrete mass and stiffness modifications for the inverse eigenstructure assignment in vibrating systems: Theory and experimental validation", International Journal of Mechanical Sciences, vol. 64, pp. 211-220, 2012.

[20] H. Ouyang, and J. Zhang, "Passive modifications for partial assignment of natural frequencies of mass-spring systems", Mechanical Systems and Signal Processing, vol.50-51, pp.214-226, 2015.

[21] Z. Liu, W. Li, H. Ouyang and D. Wang, "Eigenstructure assignment in vibrating systems based on receptances", Archive of Applied Mechanics vol.85, pp.713-724, 2015.

[22] J. Sherman, and W.J. Morrison, "Adjustment of an Inverse Matrix Corresponding to a Change in one Element of a Given Matrix", Annals of Mathematical Statistics, vol. 21(1), pp. 124-127, 1950.

[23] W.W. Hager, "Updating the Inverse of A Matrix", SIAM Review, vol. 31(2), pp.221-239, 1989.

[24] M.A. Akgün, J.H. Garcelon, and R.T. Haftka, "Fast Exact Linear and Non-Linear Structural Reanalysis and the Sherman-MorrisonWoodbury Formulas", Int. J. for Num. Meth. in Eng., vol. 50, pp. 1587-1606, 2001. 
[25] P. Level, D. Moraux, P. Drazetic, and T. Tison, "On A Direct Inversion of the Impedance Matrix in Response Reanalysis", Comm. in Num. Meth. in Eng., vol. 12, pp. 151-159, 1996.

[26] K.Y. Sanliturk, "An Efficient Method for Linear and Nonlinear Structural Modifications", Proceedings of ESDA 2002: 6th Biennial Conference on Engineering Systems Design and Analysis, ESDA 2002/APM-028, İstanbul, Türkiye, 2002.

[27] O. Çakar and K.Y. Sanliturk, "Elimination of Transducer Mass Loading Effects from Frequency Response Functions", Mechanical Systems and Signal Processing, vol. 19(1), pp. 87-104, 2005.

[28] O. Çakar, Mass and stiffness modifications without changing any specified natural frequency of a structure. Journal of Vibration and Control 17 (5), pp.769-776, 2011.

[29] M. Hüseyinoğlu and O. Çakar, "Inverse structural modification without changing a prescribed natural frequency of a mass-spring system with Multiple degree of fredoms", Proceedings of TrcIFToMM Symposium on Theory of Machines and Mechanisms, UMTS2015, TURKEY, pp.892-898, 2015, (in Turkish).

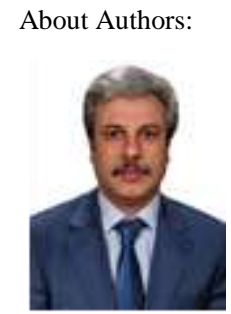

Orhan Çakar was born in Elazı ̆̆ TURKEY in 1968. $\mathrm{He}$ received his B.S. and M.S. degree in Mechanical Engineering from the University of Firat in 1990 and 1995, respectively. He received his $\mathrm{PhD}$ from Istanbul Technical University in 2003. He has served at Firat University since 1993, where he is currently associate professor. He is interested in modal testing, structural vibration, theory of machines and mechanisms and mechatronics.

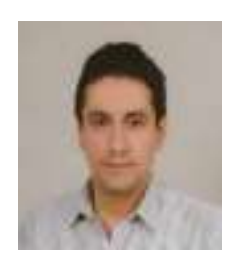

Mesut Hüseyinoğlu was born in Diyarbakır TURKEY in 1981. He received his B.S. and M.S. degree in Mechanical Engineering from the University of Firat in 2004 and 2008, respectively. He has served in Department of Mechanical Engineering at Dicle University since 2008, where he is currently research assistant, $\mathrm{He}$ is a $\mathrm{PhD}$ student at the University of Firat and he is interested in modal testing, structural vibration, theory of machines and mechanisms and mechatronics. 